\title{
Variable viscosity effects on mixed convection heat and mass transfer along a semi-infinite vertical plate in the presence of chemical reaction and viscous dissipation
}

\author{
Naikoti Kishan $^{1^{*}}$, P. Amrutha ${ }^{2}$ \\ $I^{*}$ Department of Mathematics, Osmania University, Hyderabad-7, Telangana, INDIA \\ ${ }^{2}$ St.Ann's College for Women, Mehdipatnam, Hyderabad-28, Telangana, INDIA \\ *Corresponding Author: e-mail: kishan.naikoti@gmail.com Tel +91-09346433343
}

\begin{abstract}
An analysis is carried out to study the viscous dissipation and variable viscosity effects on the flow, heat and mass transfer characteristics in a viscous fluid over a semi-infinite vertical porous plate in the presence of chemical reaction. The governing boundary layer equations are written into a dimensionless form by similarity transformations. The transformed coupled nonlinear ordinary differential equations are solved numerically by using the implicit finite difference method. The effects of different parameters on the dimensionless velocity, temperature, and concentration profiles are shown graphically. In addition, tabulated results for the local skin-friction coefficient, the local Nusselt number, and the local Sherwood number are presented and discussed.
\end{abstract}

Keywords: Variable viscosity, Chemical Reaction, Viscous Dissipation, Finite difference method, Suction.

DOI: http://dx.doi.org/10.4314/ijest.v7i2.3

\section{Introduction}

In nature, the presence of pure air or water is impossible. Some foreign mass may be present either naturally or mixed with the air or water. A large amount of research work has been reported in this field. In particular, the study of chemical reaction, heat and mass transfer with heat source is of considerable importance in chemical and hydrometallurgical industries. Chemical reaction can be codified as either heterogeneous or homogeneous processes. This depends on whether they occur at an interface or as a single phase volume reaction.

The study of heat and mass transfer with chemical reaction is of great practical importance to engineers and scientists because of its almost universal occurrence in many branches of science and engineering. The flow of a fluid past a wedge is of fundamental importance since this type of flow constitutes a general and wide class of flows in which the free stream velocity is proportional to a power of the length coordinate measured from the stagnation point. All industrial chemical processes are designed to transform cheaper raw materials to high value products (usually via chemical reaction). A reactor, in which such chemical transformations take place, has to carry out several functions like bringing reactants into intimate contact, providing an appropriate environment (temperature and concentration fields) for adequate time and allowing for removal of products. Fluid dynamics plays a pivotal role in establishing relationship between reactor hardware and reactor performance.

In the studies on heat transfer in the saturated porous media, the thermo physical properties of the fluid are assumed to be constant; however, it is know that these properties may change with temperature, especially for fluid viscosity. For most realistic fluids, the viscosity shows a rather pronounced variation with temperature. To accurately predict the heat transfer rate, it is necessary to take into account this variation of viscosity. The effect of temperature-dependent viscosity on the mixed convection flow from vertical plate is investigated by several authors Das et.al (2008), Hunegnaw and Kishan (2014), Kafoussius \& Williams (1995), Kafoussius et al. (1998) and Lai and Kulacki (1990). Pop et al. (1992) and Hassanien (1997) made an attempt to examine 
the effect of variable viscosity on the flow and heat transfer on continuous linearly stretching surface and obtained the similarity solutions for an isothermally heated plate with fluid viscosity varied as an inverse function of temperature. Further Hossain et al (2002) studied the natural convection of fluid with variable viscosity from a heated vertical wavy surface. The variable viscosity effects on non-Darcy free or mixed convection flow on a vertical surface in a fluid saturated porous medium is studied by Jayanthi and Kumari (2007). An analysis is carried out to study the variable viscosity and chemical reaction effects on flow, heat and mass transfer characteristics in a viscous fluid over a porous wedge in the presence of heat radiation by Kandasamy, et al. (2007). The Falkner-Skan flow with constant wall temperature and variable viscosity is studied by Pantokratoras (2006).

Kandasamy et al. (2009) analyzed the effects of thermophoresis and variable viscosity on MHD mixed convective heat and mass transfer of a viscous, incompressible and electrically conducting fluid past a porous wedge in the presence of chemical reaction The unsteady mixed convection boundary layer flow near the region of a stagnation point on a vertical surface embedded in a Darcian fluid-saturated porous medium is studied by Roslinda et al. (2004). The mixed convection boundary layer flow through a stable stratified porous medium bounded by a vertical surface is investigated by Anuar et al. (2008). Hassanien and Al-arabi (2009) studied the unsteady mixed convection boundary layer flow near the stagnation point on a heated vertical plate embedded in a fluid saturated porous medium. The effect of temperature dependent viscosity on a laminar mixed convection boundary layer flow and heat transfer on continuous moving vertical surface is studied by Mohamed and Ali (2006).

Hossian (1992) analyzed the viscous and Joule heating effects on MHD free convection flow with variable plate temperature. Hakiem et al. (1999) studied the Joule heating effects on MHD free convection flow of a micro polar fluid. Mostafa and Mahmoud (2007) studied the effects of chemical reaction and variable viscosity on flow, heat and mass transfer on a semi-infinite vertical plate with suction. The order of chemical reaction in this work is taken as first-order reaction. The aim of present work is to study the effects of viscous dissipation and variable viscosity on the flow of heat and mass transfer characteristics in a viscous fluid over a semi-infinite vertical porous plate in the presence of chemical reaction. The model problem is obtained and solved numerically using a implicit finite difference scheme coupled with a Thomas algorithm. Pertinent results are presented graphically and discussed quantitatively, and also tabulated results for the local skin-friction coefficient, the local Nusselt number, and the local Sherwood number.

\section{Mathematical Analysis}

Consider a steady, viscous incompressible Newtonian fluid past a semi-infinite vertical porous plate which is aligned parallel to a uniform free stream with velocity $u_{\infty}$. It is assumed that the fluid has constant properties except the density in the buoyancy term of the momentum equation and in the fluid viscosity which is assumed to be an inverse linear function of temperature (Lai and Kulacki 1990).

Under the above assumptions and Boussinesq's approximation, the boundary layer equations governing the flow can be expressed as (Pop.I and Ingham 2001)

$$
\begin{aligned}
& \frac{\partial u}{\partial x}+\frac{\partial v}{\partial y}=0 \\
& u \frac{\partial u}{\partial x}+v \frac{\partial u}{\partial y}=\frac{1}{\rho_{\infty}} \frac{\partial}{\partial y}\left(\mu \frac{\partial u}{\partial y}\right)+g \beta\left(T-T_{\infty}\right)+g \beta *\left(C-C_{\infty}\right) \\
& u \frac{\partial T}{\partial x}+v \frac{\partial T}{\partial y}=k \frac{\partial^{2} T}{\partial y^{2}}+\mu\left(\frac{\partial u}{\partial y}\right)^{2} \\
& u \frac{\partial C}{\partial x}+v \frac{\partial C}{\partial y}=D \frac{\partial^{2} C}{\partial y^{2}}-K_{1}\left(C-C_{\infty}\right)
\end{aligned}
$$

The appropriate boundary conditions are

$$
\begin{aligned}
& \text { At } \quad y=0 ; \quad u=0, \quad v=-v_{w}, \quad C=C_{\infty}, \quad T=T_{w} \\
& \text { As } \quad y \rightarrow \infty ; \quad u \rightarrow u_{\infty}, \quad C \rightarrow C_{\infty}, \quad T \rightarrow T_{\infty}
\end{aligned}
$$

where the $x$-axis is taken along the plate in the vertically upward direction and the $y$-axis is taken normally to the plate. Here, $u$ and $v$ are the velocity components along $x$ and $y$, respectively. $T$ is the fluid temperature, $C$ is the species concentration, $T_{\infty}$ is the

ambient temperature, $C_{\infty}$ is the ambient concentration, $\rho_{\infty}$ is the ambient density, $g$ is the gravitational acceleration, $\beta$ is the 
coefficient of thermal expansion, $\beta^{*}$ is the coefficient of concentration expansion, $\mu_{\infty}$ is the ambient viscosity, $\mu$ is the viscosity of the fluid, $k$ is the thermal diffusivity, $D$ is the molecular diffusion coefficient, $K_{1}$ is the chemical reaction parameter, and $v_{w}$ $(>0)$ is the suction velocity.

Introducing the similarity variables as

$$
\eta=\sqrt{\frac{u_{\infty}}{v_{\infty} x}} y \quad ; \quad \psi(x, y)=\sqrt{v_{\infty} u_{\infty} x} f(\eta)
$$

The velocity components are given by

$$
u=\frac{\partial \psi}{\partial y}, \quad v=-\frac{\partial \psi}{\partial x}
$$

It can be easily verified that the continuity equation (1) is identically satisfied. Equations (2) - (4) reduce to

$$
\begin{gathered}
f^{\prime \prime \prime}+\frac{1}{2}\left(\frac{\theta_{r}-\theta}{\theta_{r}}\right) f f^{\prime \prime}+\frac{\theta^{\prime}}{\left(\theta_{r}-\theta\right)} f^{\prime \prime}+\frac{G r}{\operatorname{Re}^{2}}\left(\frac{\theta_{r}-\theta}{\theta_{r}}\right) \theta+\frac{G c}{\operatorname{Re}^{2}}\left(\frac{\theta_{r}-\theta}{\theta_{r}}\right) \phi=0 \\
\theta^{\prime \prime}+\frac{1}{2} \operatorname{Pr} f \theta^{\prime}+E c\left(f^{\prime \prime}\right)^{2}=0 \\
\phi^{\prime \prime}+\frac{1}{2} S c f \phi^{\prime}-\gamma S c \operatorname{Re} \phi=0
\end{gathered}
$$

where $\theta=\frac{\left(T-T_{\infty}\right)}{\left(T_{w}-T_{\infty}\right)} ; \phi=\frac{\left(C-C_{\infty}\right)}{\left(C_{w}-C_{\infty}\right)} ; \theta_{r}=-\frac{1}{\alpha\left(T_{w}-T_{\infty}\right)}$ is the viscosity parameter, $\operatorname{Re}=\frac{u_{\infty} x}{v_{\infty}}$ is the local Reynolds number, $\quad E c=\frac{\mu u_{\infty}^{2}}{k\left(T_{w}-T_{\infty}\right)} \quad$ is the Eckert number, $\quad G r=g \beta \frac{\left(T_{w}-T_{\infty}\right) x^{3}}{v_{\infty}^{2}} \quad$ is the local Grashof number, $G c=g \beta^{*} \frac{\left(C_{w}-C_{\infty}\right) x^{3}}{v_{\infty}^{2}}$ is the local modified Grashof number, $\gamma=K_{1} \frac{v_{\infty}}{u_{\infty}^{2}}$ is the chemical reaction parameter, $\operatorname{Pr}=\frac{\mu c_{p}}{k}$ is the Prandtl number, $S c=\frac{v_{\infty}}{D}$ is the Schmidt number, $\alpha$ is a constant ( $>0$ for liquids) and $\left(<0\right.$ for gases), $v_{\infty}=\frac{\mu_{\infty}}{\rho_{\infty}}, c_{p}$ is the specific heat at constant pressure, and the primes denote differentiation with respect to $\eta$.

The transformed boundary conditions are given by

$$
\begin{aligned}
& \eta=0 ; \quad f=f_{w}, \quad f^{\prime}=0, \quad \theta=1, \quad \phi=1, \\
& \eta \rightarrow \infty ; \quad f^{\prime} \rightarrow 1, \quad \theta \rightarrow 0, \quad \phi \rightarrow 0
\end{aligned}
$$

where $f_{w}=-\sqrt{\frac{x}{v_{\infty} u_{\infty}}} v_{w}$ (suction parameter $\left.>0\right)$.

The physical quantities of interest in this problem are the local skin-friction coefficient $C_{f}$, the local Nusselt number $N u$, and the local Sherwood number $S h$ which are defined by

$$
C_{f}=\frac{2 \theta_{r}}{\left(\theta_{r}-1\right)} \operatorname{Re}^{-\frac{1}{2}} f^{\prime \prime}\left(0, \theta_{r}\right) ; N u=-\operatorname{Re}^{\frac{1}{2}} \theta^{\prime}\left(0, \theta_{r}\right) ; S h=-\operatorname{Re}^{\frac{1}{2}} \phi^{\prime}\left(0, \theta_{r}\right)
$$

The equation (8) can be Re-written as

$$
f^{\prime \prime \prime}+A[i] f f^{\prime \prime}+B[i] f^{\prime \prime}+C[i] \theta[i]+C[i] \phi[i]=0
$$

Quasi-linearization of all highly non-linear terms (Bellman \& Kalaba, 1965), the above equation gives

$$
f^{\prime \prime \prime}+A[i] F f^{\prime \prime}+A[i] F^{\prime \prime} f+B[i] f^{\prime \prime}-A[i] F F^{\prime \prime}+C[i] \theta[i]+C[i] \phi[i]=0
$$


where $A[i]=\frac{1}{2}\left(\frac{\theta_{r}-\theta}{\theta_{r}}\right) ; B[i]=\frac{\theta^{\prime}}{\theta_{r}-\theta} \quad$ and $\quad C[i]=\frac{G r}{\operatorname{Re}^{2}}\left(\frac{\theta_{r}-\theta}{\theta_{r}}\right)$

Here $F$ is assumed to be known function, i.e.. $F$ is the $n^{\text {th }}$ approximation to the solution and $f$ is the $(n+1)^{\text {th }}$ approximation to the solution. Using implicit finite difference scheme, the equations (13), (9) and (10) are transformed to

$$
\begin{aligned}
& a 2[i] f[i+1]+b 2[i] f[i]+c 2[i] f[i-1]+f[i-2]=d 2[i] \\
& a[i] \phi[i-1]-b[i] \phi[i]+c[i] \phi[i+1]=0 \\
& a 1[i] \theta[i-1]-b[i] \theta[i]+c 1[i] \theta[i+1]+d 1[i]=0
\end{aligned}
$$

where

$$
\begin{array}{llr}
a[i]=1-(0.25 h S c f[i]) & b[i]=2+\left(h^{2} S c \operatorname{Re} \gamma\right) & c[i]=1+(0.25 h S c f[i]) \\
a 1[i]=1-(0.25 h \operatorname{Pr} f[i]) & b 1[i]=2 & c 1[i]=1+(0.25 h \operatorname{Pr} f[i]) \\
d 1[i]=h^{2} E c F 2[i] F 2[i] & a 2[i]=(A[i] F[i]+B[i]) h-3 & \\
b 2[i]=\left(3+h^{3} A[i] F 2[i]-2 h(A[i] F[i]+B[i])\right. & c 2[i]=(A[i] F[i]+B[i]) h-1 \\
d 2[i]=h^{3}((A[i] F[i] F 2[i]-C[i](\theta[i]+\phi[i])) &
\end{array}
$$

Here $\mathrm{h}$ represents the mesh size taken as $\mathrm{h}=0.02$. The system of equations (14), (15) and (16) have been solved by GaussSeidel iteration method and numerical values are carried out after executing the computer program for it. In order to prove convergence of finite-difference scheme, the computations are carried out for different values of $\mathrm{h}$ by running the same programme. Negligible change is observed, after each cycle of iteration the tolerance set as $10^{-4}$ is satisfied at all points.

\section{Results and Discussion}

Equations (8)-(10) with the boundary conditions (11) were solved numerically. First, Quasi- linearization technique can be applied to linearize the momentum equation (8). The linearized coupled ordinary differential equations (9)-(10) and (13) with boundary conditions (11) are solved by using the implicit finite difference method. To verify the accuracy of the numerical method the obtained results $f^{\prime \prime}(0)$ and $-\theta^{\prime}(0)$ are checked against previously published work those reported by Mostafa and Mahmoud (2007) for special cases of the problem and are found to be in good agreement. In the present study our results for $f^{\prime \prime}(0)$ and $-\theta^{\prime}(0)$ for $\mathrm{Gr}=0, \mathrm{Gc}=0, \theta_{r}=0.2$, and $\operatorname{Pr}=0.7$ are $-0.19432,0.26958$, respectively which are exactly as reported in Mostafa and Mahmoud (2007).

The velocity, temperature and concentration profiles obtained in the dimensionless form are presented in figures $1-17$ for $\operatorname{Pr}=$ 0.7 which represent air at temperature $20^{\circ} \mathrm{C}$ and for $\mathrm{Sc}=0.6$ which corresponds to water vapor that represents a diffusion chemical species of most common interest in air. The values for Grashof number for heat transfer is chosen to be $\mathrm{Gr}=5$ and modified Grashof number for mass transfer is chosen to be $\mathrm{Gc}=4$, since these values correspond to a cooling problem. The values of $\gamma$ is chosen to be $0.5,1,3$, and the values of $f_{w}$ are chosen to be $0.3,0.5$, and 1 . It is important to note that $\theta_{r}$ is negative for liquids and positive for gases when $T_{w}-T_{\infty}$ is positive. The values of $\theta_{r}$ (for air $\theta_{r}>0$ ) are chosen to be 2, 6, and 10 


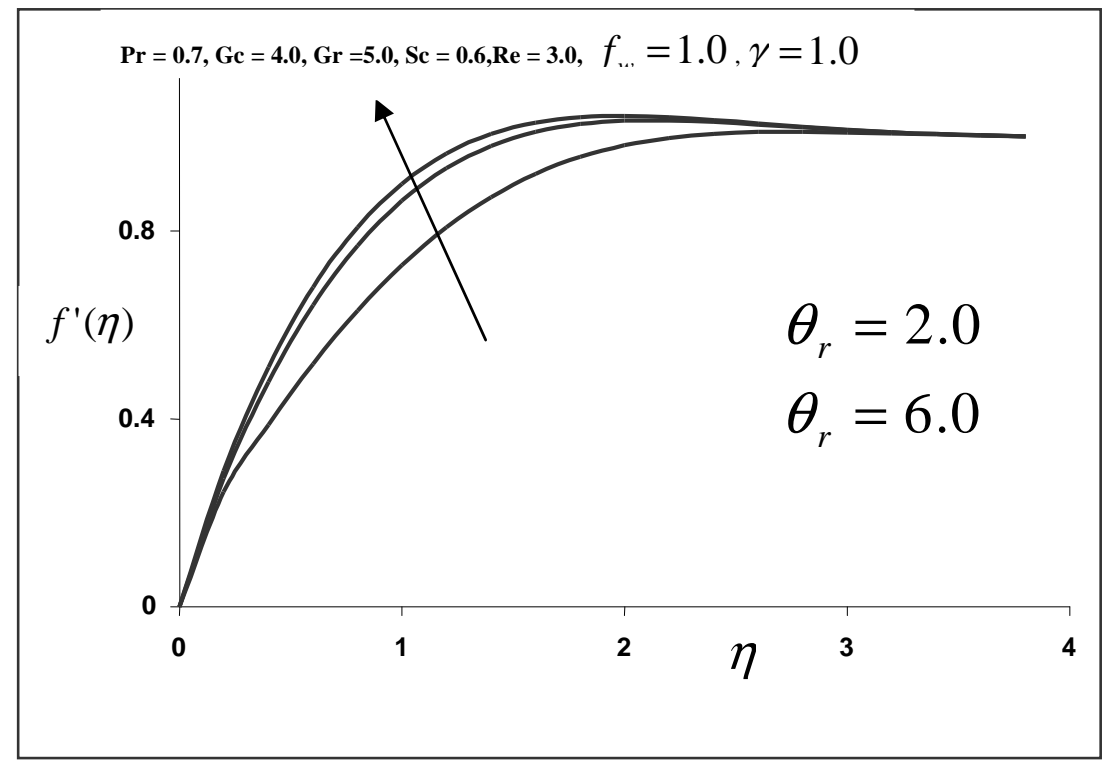

Figure 1 Velocity profiles for different values of viscosity temperature parameter

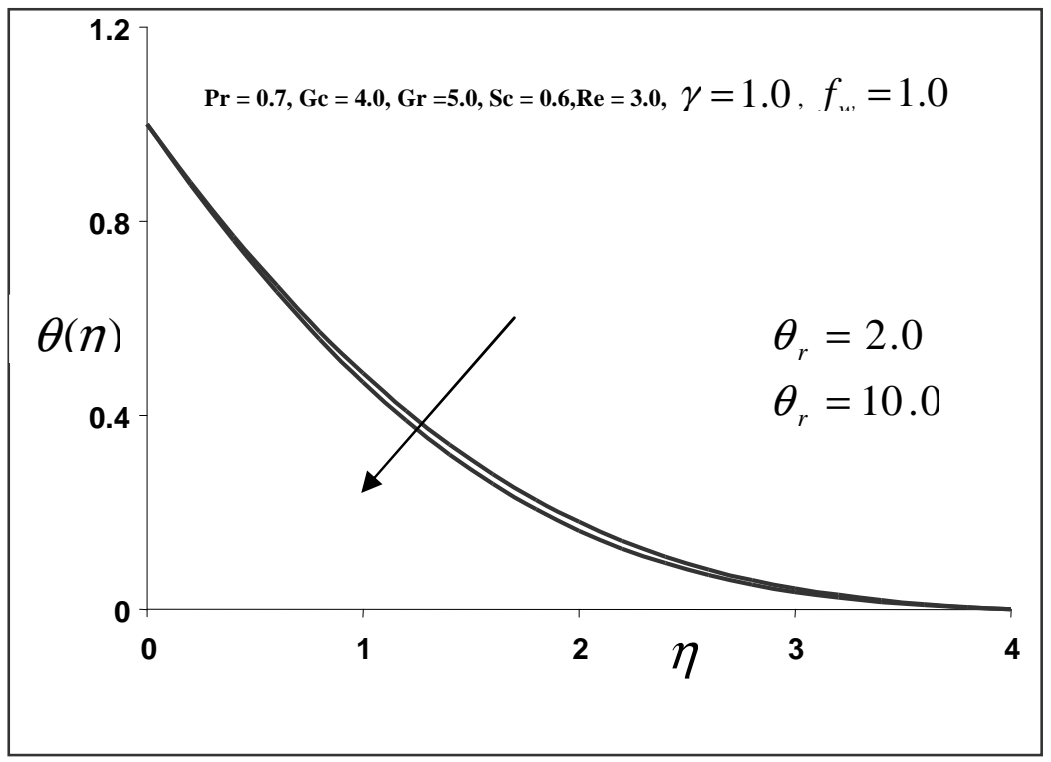

Figure 2 Temperature profiles for different values of viscosity temperature parameter

Figures 1-3 illustrate the effects of the viscosity parameter $\theta_{r}$ on the velocity, temperature, and concentration profiles respectively. It is seen from the figures that the velocity increases with the increase of the viscosity parameter, while the thermal boundary layer thickness decreases as the viscosity parameter increases. So, the increase of viscosity parameter accelerates the fluid motion and reduces the temperature of the fluid along the wall. Also, one sees that the concentration of the fluid is almost not affected with increase of the viscosity parameter.

Figures 4-6 portray the influence of the chemical reaction parameter $\gamma$ on the velocity, temperature, and concentration profiles respectively. It is clear from the figures that increasing the values of $\gamma$ produces a decrease in the velocity. This means that in the case of suction, the chemical reaction decelerates the fluid motion while the temperature of the fluid is almost not affected with increase of $\gamma$. Also, the chemical reaction decelerates the concentration of the fluid in the case of suction. 


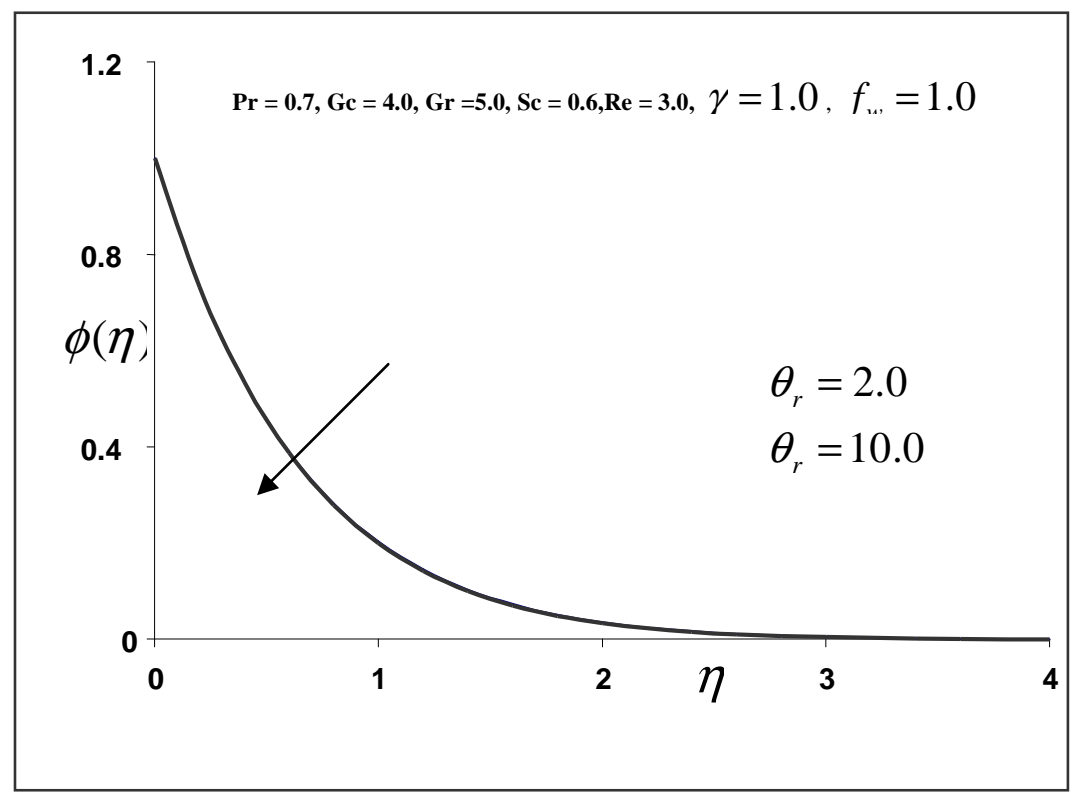

Figure 3 Concentration profiles for different values of viscosity temperature parameter $\theta_{r}$

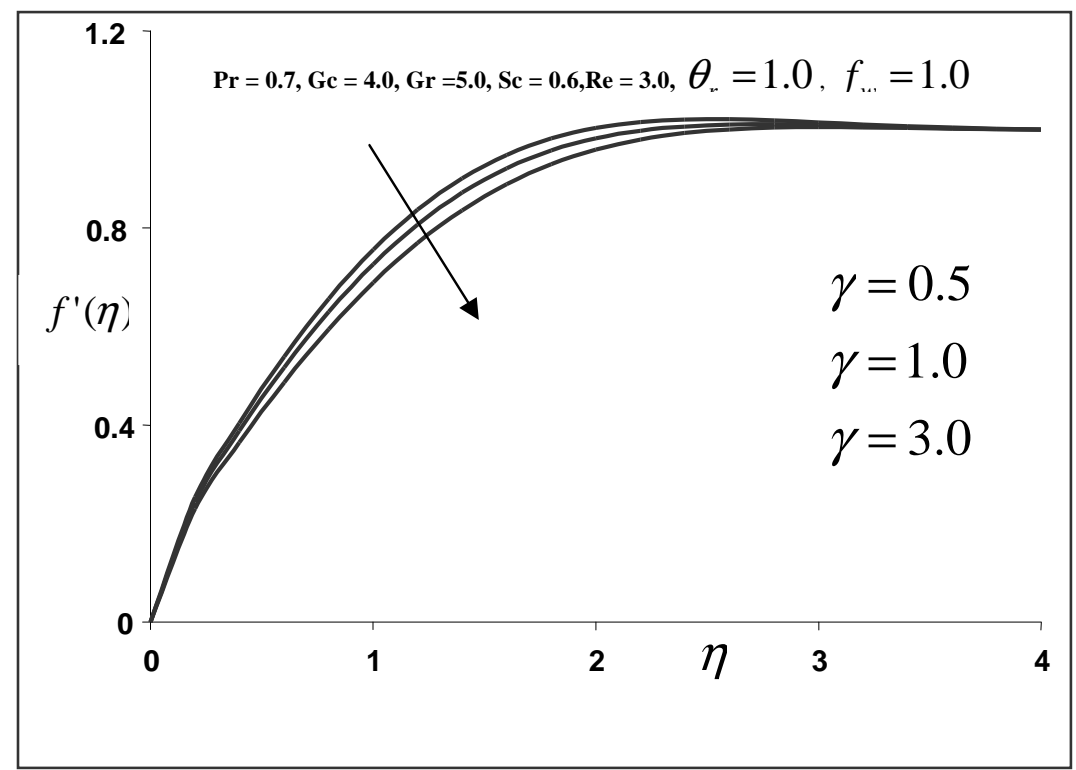

Figure 4 Velocity profiles for different values of chemical reaction parameter $\gamma$

Figures 7-9 reflect the influence of the suction parameter $f_{w}$ on the velocity, temperature and concentration profiles in the boundary layer. From the figures one sees that the thickness of the velocity boundary layer increases as the suction parameter increases so velocity decreases. In the same way the thickness of the temperature boundary layer and the thickness of the concentration boundary layer decrease with an increase in the suction parameter $f_{w}$. Figs.10 and 11 demonstrate the dimensionless velocity and temperature profiles for different values of Grashof number Gr. From the figure it is clear that the velocity of the fluid increases with increase of Grashof number Gr, and the temperature of the fluid decreases with increase in Gr.

Figures 12-14 reflects the velocity, temperature and concentration profiles respectively, for different values of Reynolds number Re. From the figures it is observed that the velocity and the concentration of the fluid decreases with increase of Re, while the temperature of the fluid increases with the increase of Re. Figures 15-17 show the dimensionless velocity, temperature and concentration profiles respectively, for different values of Schmidt number Sc. From the figures it is seen that the velocity and temperature of the fluid increases with the increase of Sc, where as the concentration of the fluid decreases with increase in Sc. Figs.18-20 display dimensionless velocity, temperature and concentration profiles respectively, for different values of Eckert 
number Ec. From the figures it is seen that the velocity of the fluid decrease with increase of Eckert number Ec, where as the temperature and concentration of the fluid increases with increase in Eckert number Ec.

Table 1 Effects of various parameters on $f^{\prime \prime}(0),-\theta^{\prime}(0)$ and $-\phi^{\prime}(0)$ with $R e=3.0$.

\begin{tabular}{lrrrccc}
\hline$E c$ & $\theta_{r}$ & $\gamma$ & $f_{w}$ & $f^{\prime}(0)$ & $-\theta^{\prime}(0)$ & $-\phi^{\prime}(0)$ \\
\hline 0 & 2 & 1 & 0.5 & 0.72645 & 0.50845 & 1.43342 \\
& 6 & 1 & 0.5 & 1.12446 & 0.52776 & 1.43817 \\
& 10 & 1 & 0.5 & 1.19989 & 0.53078 & 1.43845 \\
& 2 & 0.5 & 0.5 & 0.75413 & 0.51107 & 1.05736 \\
& 2 & 1 & 0.5 & 0.72649 & 0.50878 & 1.43325 \\
& 2 & 3 & 0.5 & 0.68432 & 0.50583 & 2.40448 \\
& 2 & 1 & 0.3 & 0.70174 & 0.46461 & 1.40134 \\
& 2 & 1 & 0.5 & 0.72678 & 0.50945 & 1.43293 \\
& 2 & 1 & 1 & 0.79382 & 0.62884 & 1.51636 \\
& & & & & & \\
0.01 & 2 & 1 & 0.5 & 0.72450 & 0.51649 & 1.44624 \\
& 6 & 1 & 0.5 & 1.12124 & 0.53678 & 1.44695 \\
& 10 & 1 & 0.5 & 1.19685 & 0.54123 & 1.44964 \\
& & & & & & \\
0.1 & 2 & 1 & 0.5 & 0.72443 & 0.52359 & 1.45514 \\
& 6 & 1 & 0.5 & 1.12068 & 0.54469 & 1.45915 \\
& 10 & 1 & 0.5 & 1.19588 & 0.54886 & 1.46064 \\
& & & & & & \\
\hline
\end{tabular}

Table 1 represents the effects of $\theta_{r}, \gamma$, and $f_{w}$ on the values of the skin-friction coefficient $f^{\prime \prime}(0)$, the Nusselt number $-\theta^{\prime}(0)$, and the Sherwood number $-\phi^{\prime}(0)$ for different Ec values. The results show that the skin-friction coefficient, the Nusselt number and the Sherwood number increase as $\theta_{r}$ or $f_{w}$ increases. Also, as the chemical parameter $\gamma$ increases, the skin-friction coefficient and the Nusselt number decrease, while the Sherwood number increases. It can be seen that the Nusselt number and the Sherwood number increase as Ec increases, while the skin-friction coefficient decreases as Ec increases.

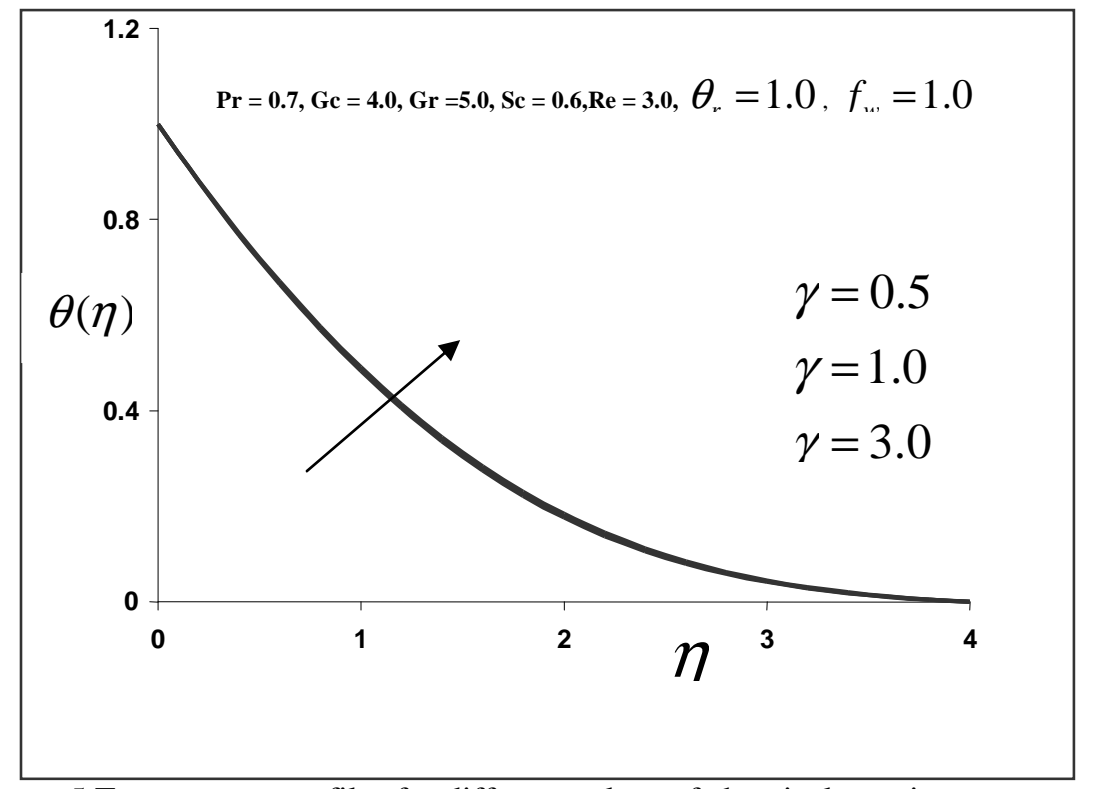

Figure 5 Temperature profiles for different values of chemical reaction parameter $\gamma$ 


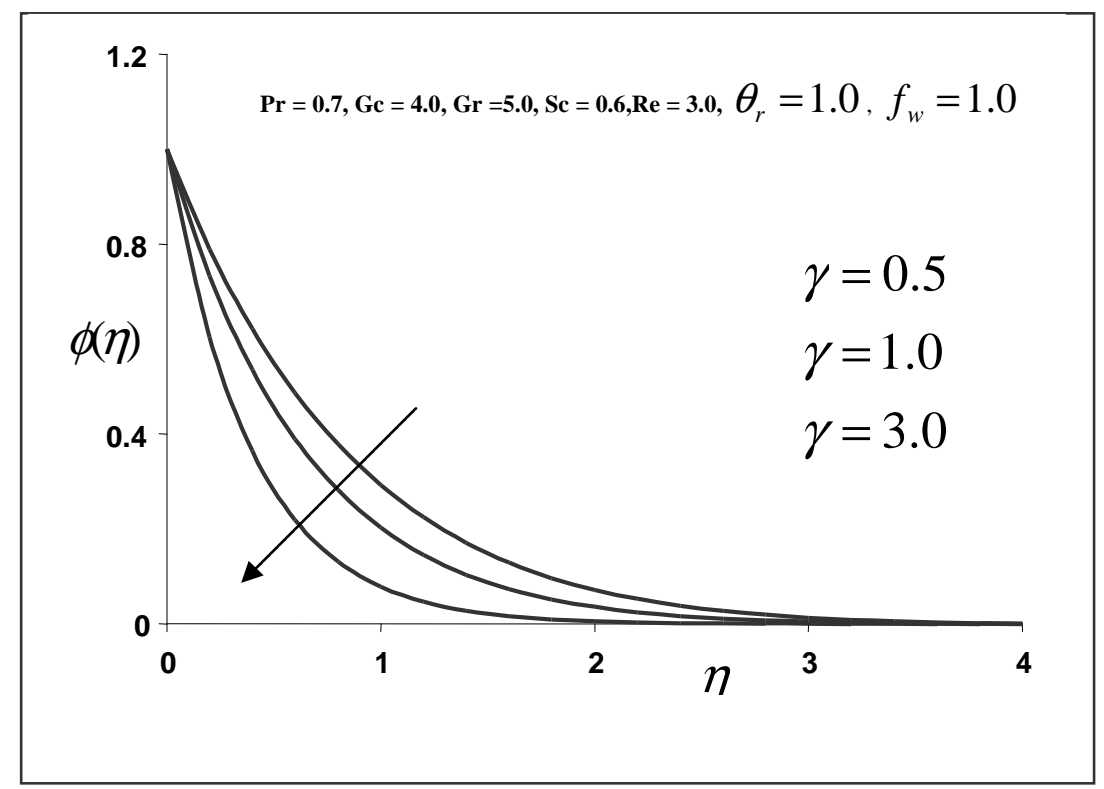

Figure 6 Concentration profiles for different values of chemical reaction parameter

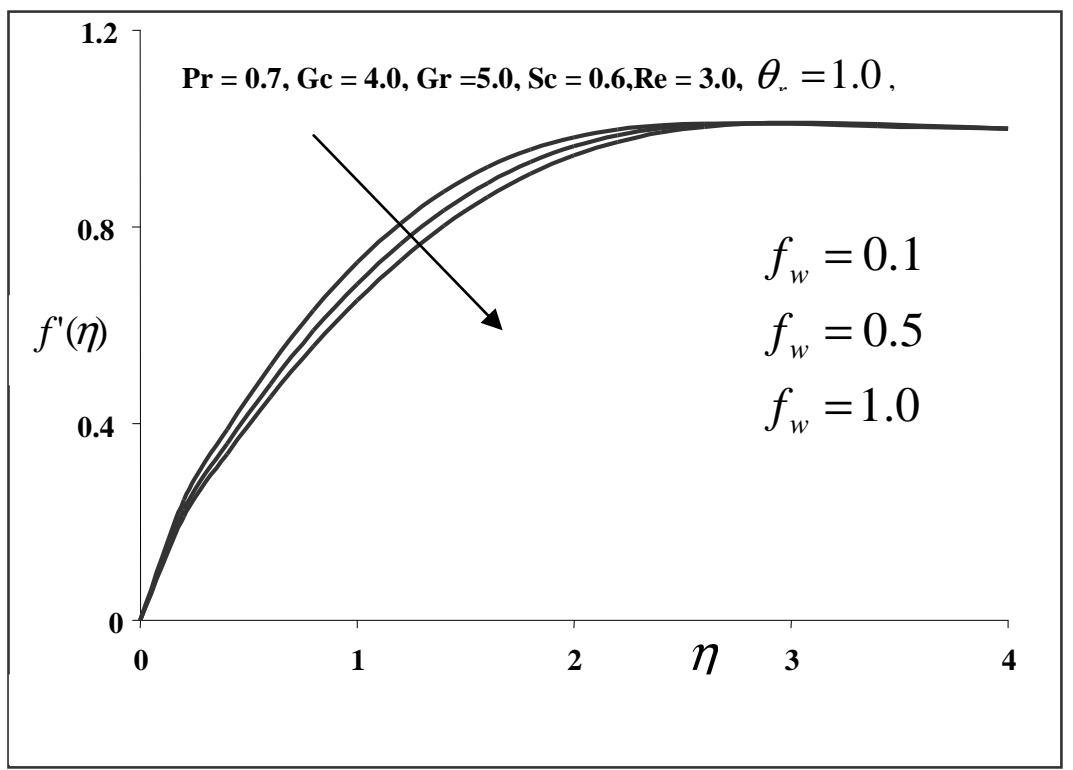

Figure 7 Velocity profiles for different values of suction parameter $f_{w}$ 


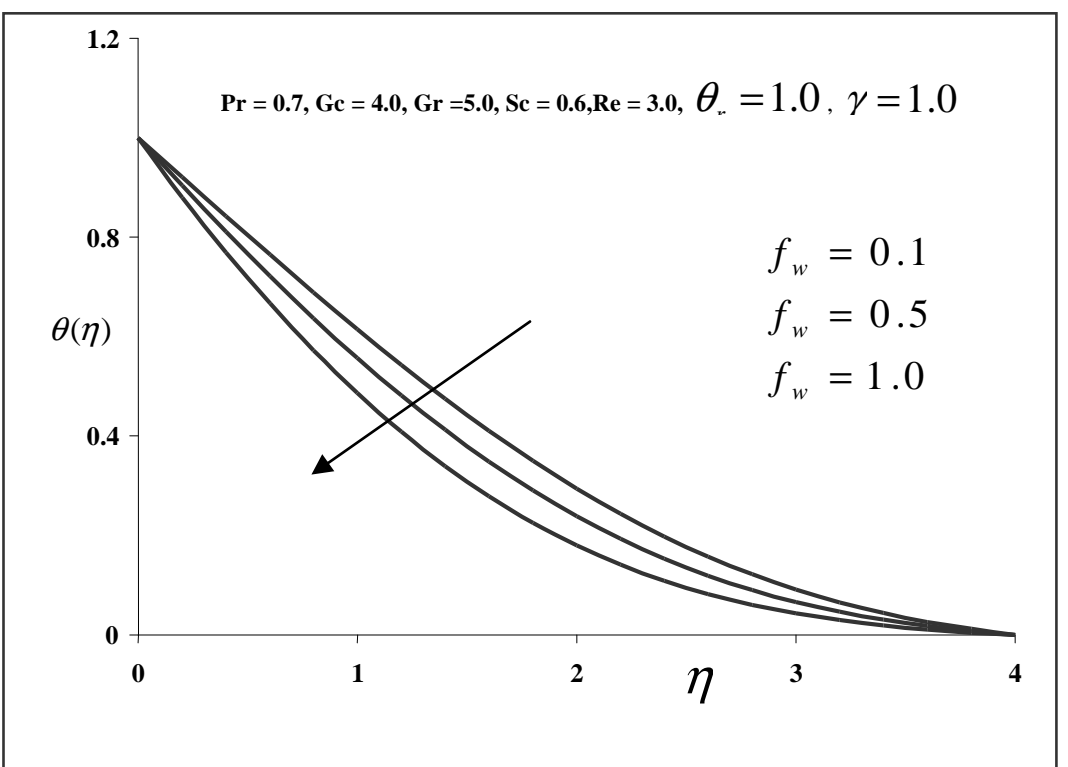

Figure 8 Temperature profiles for different values of suction parameter $f_{w}$

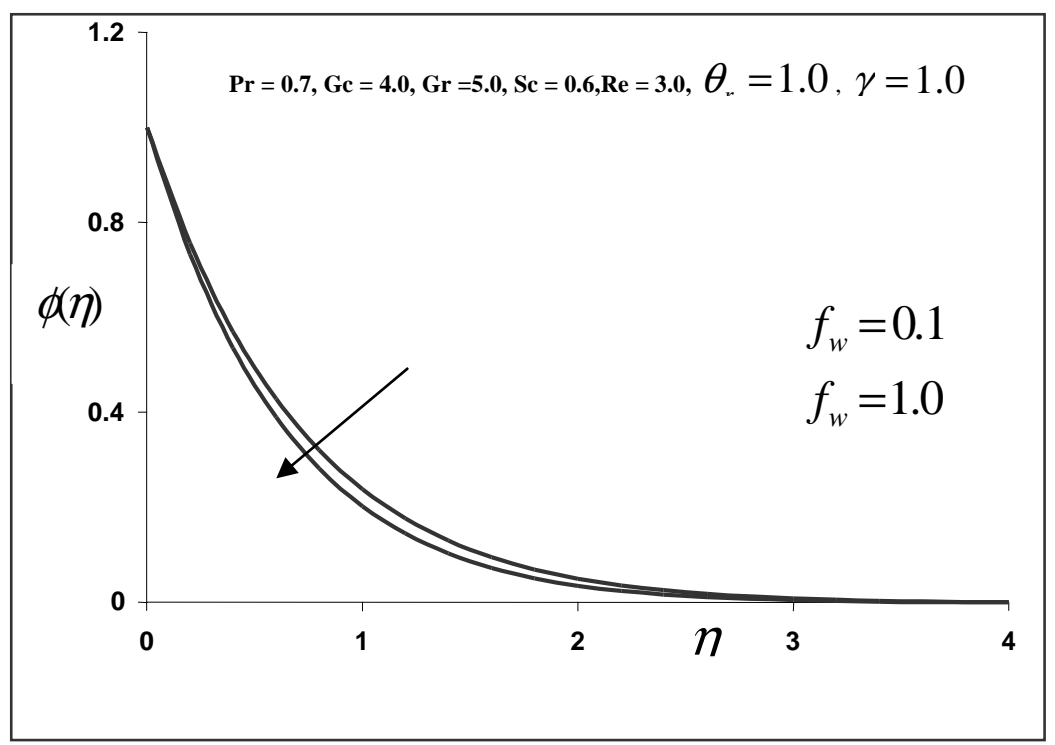

Figure 9 Concentration profiles for different values of suction parameter $f_{w}$ 


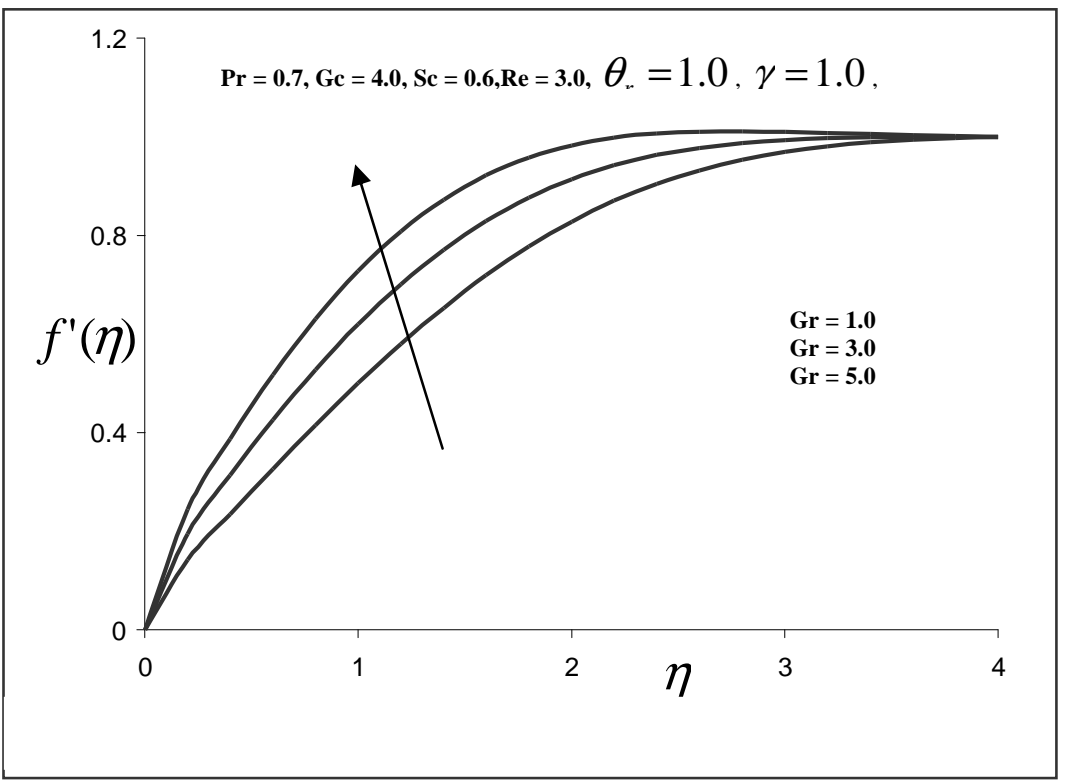

Figure 10 Velocity profiles for different values of Grashof number

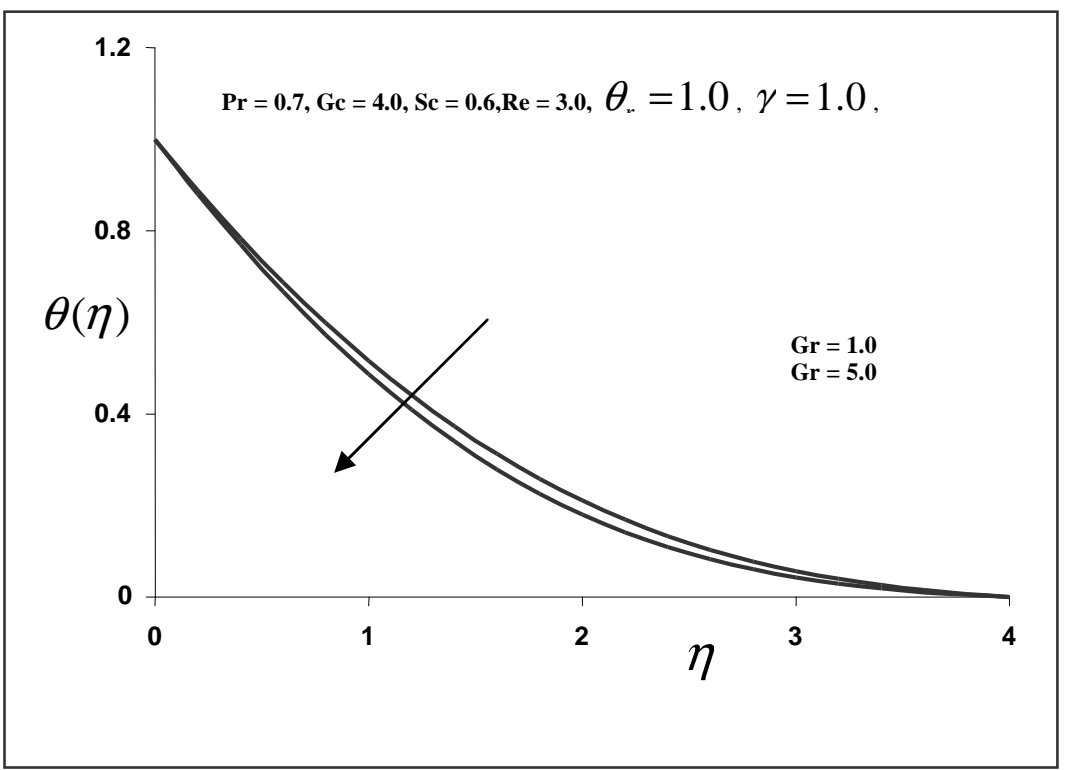

Figure 11 Temperature profiles for different values of Grashof number 


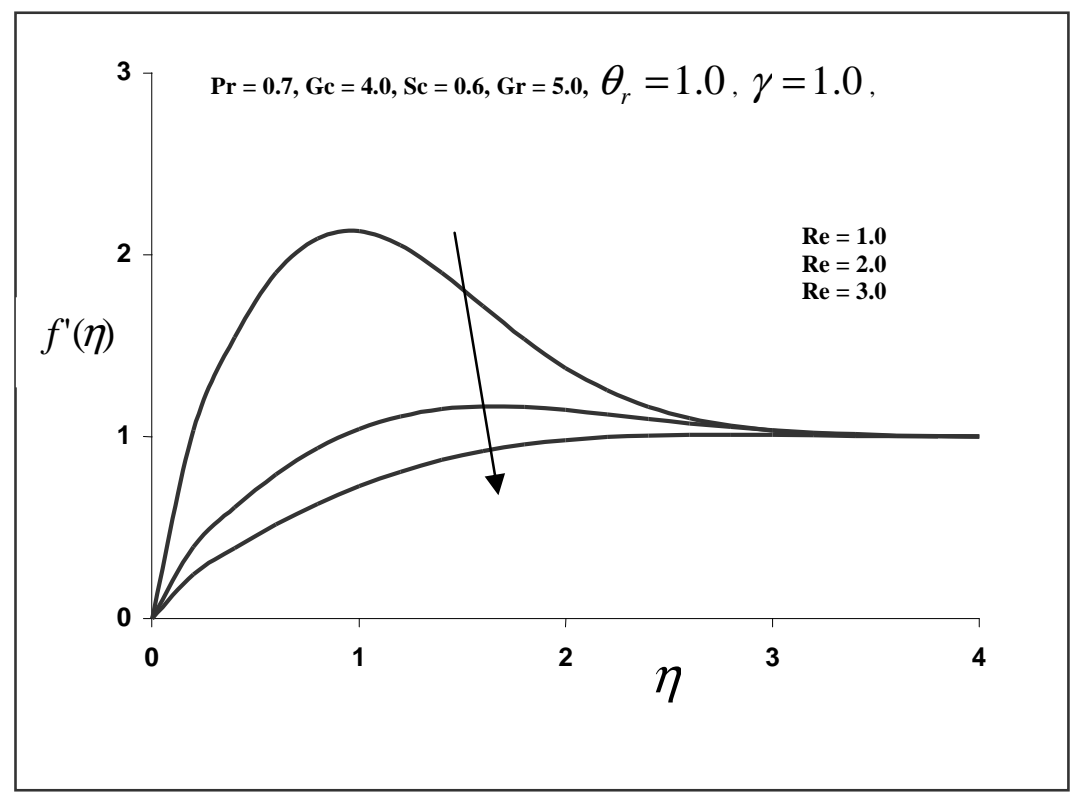

Figure 12 Velocity profiles for different values of Reynolds number Re

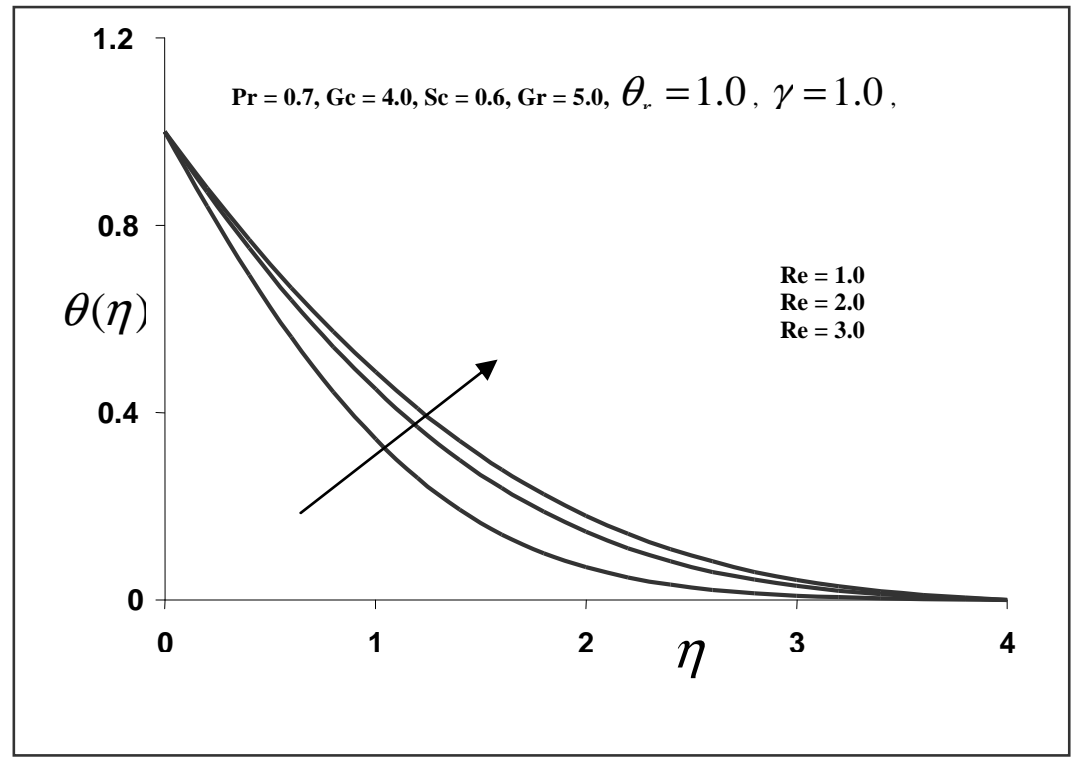

Figure 13 Temperature profiles for different values of Reynolds Number, Re 


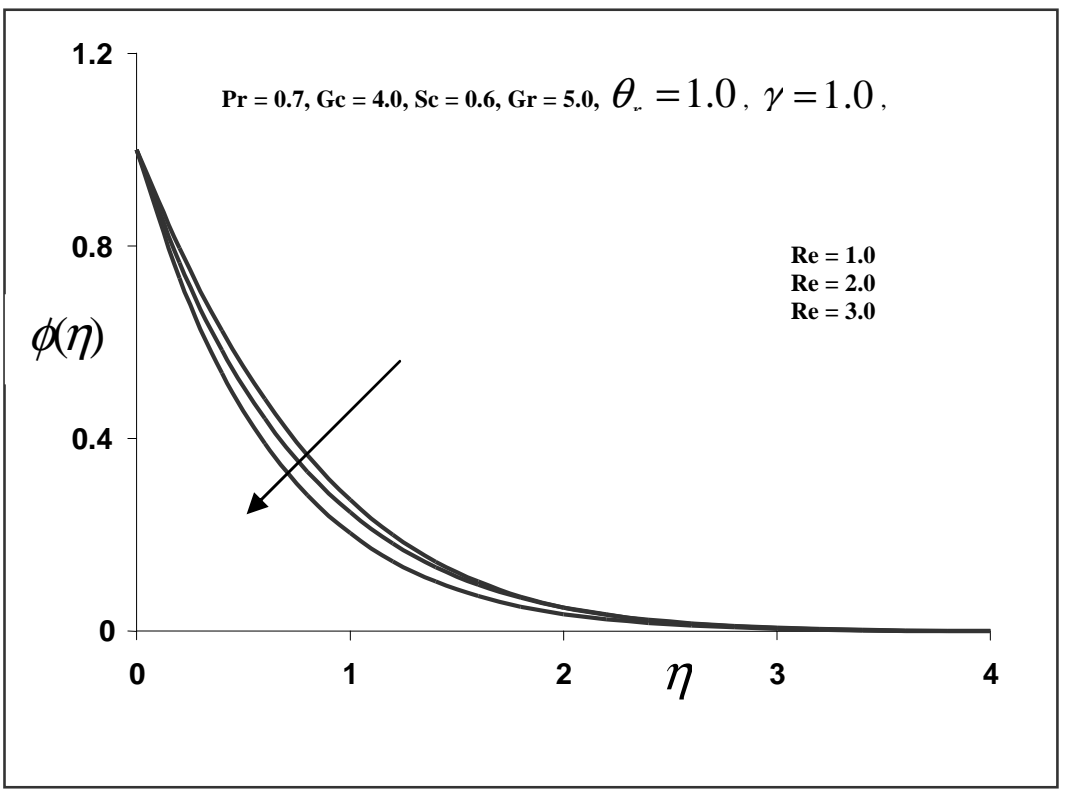

Figure 14 Concentration profiles for different values of Reynolds number Re

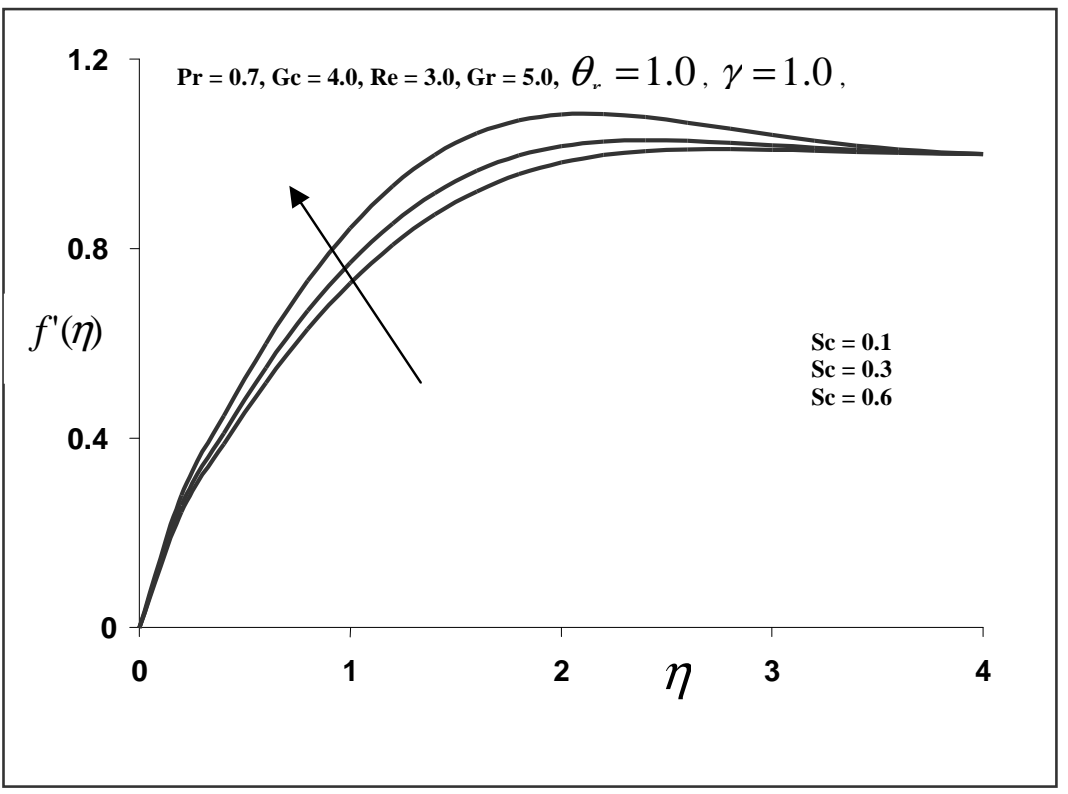

Figure 15 Velocity profiles for different values of Schmidt number Sc 


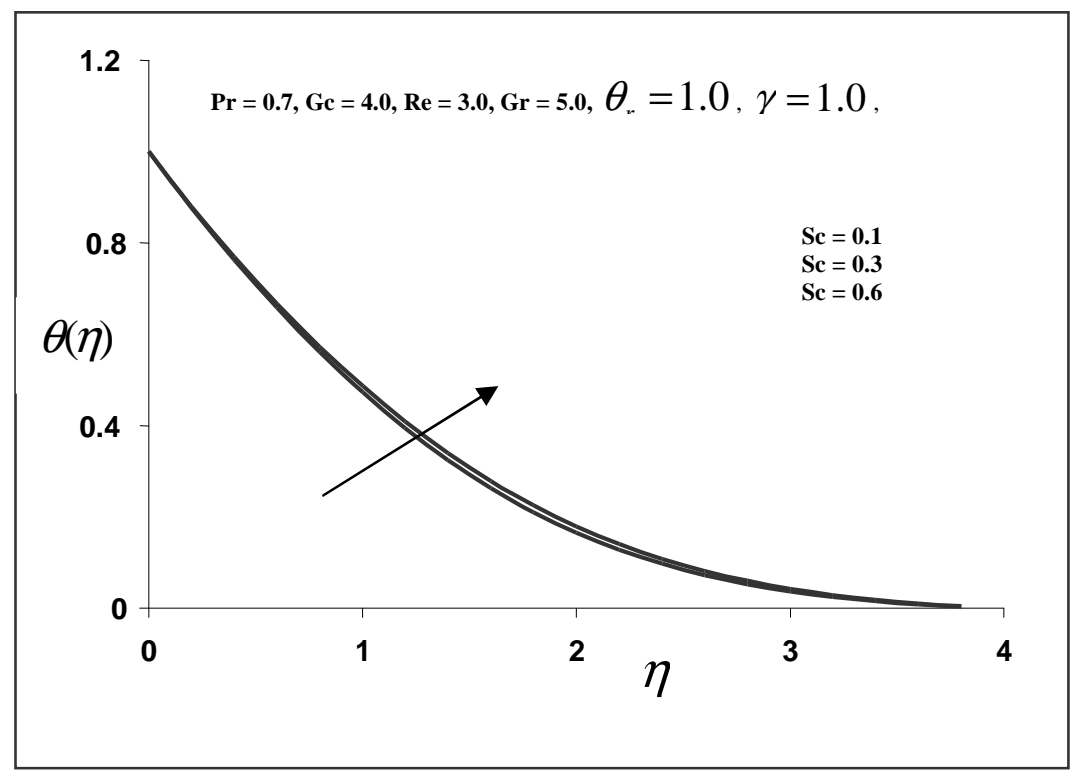

Figure 16 Temperature profiles for different values of Schmidt number Sc

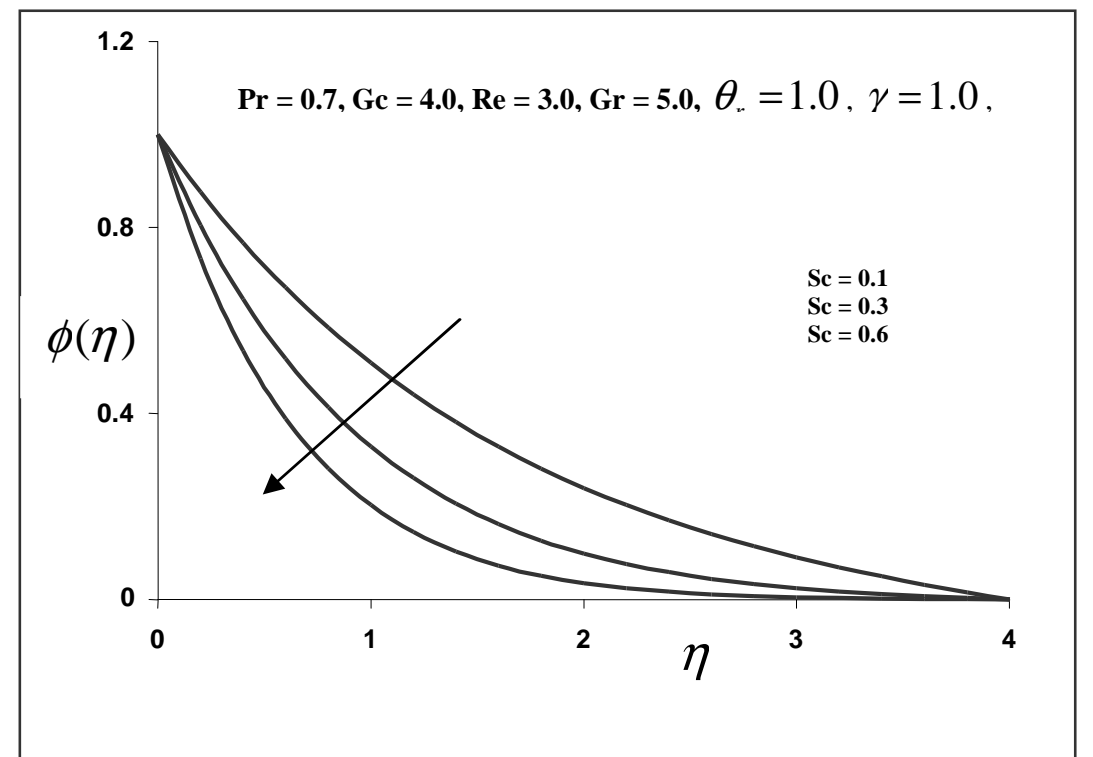

Figure 17 Concentration profiles for different values of Schmidt number Sc 


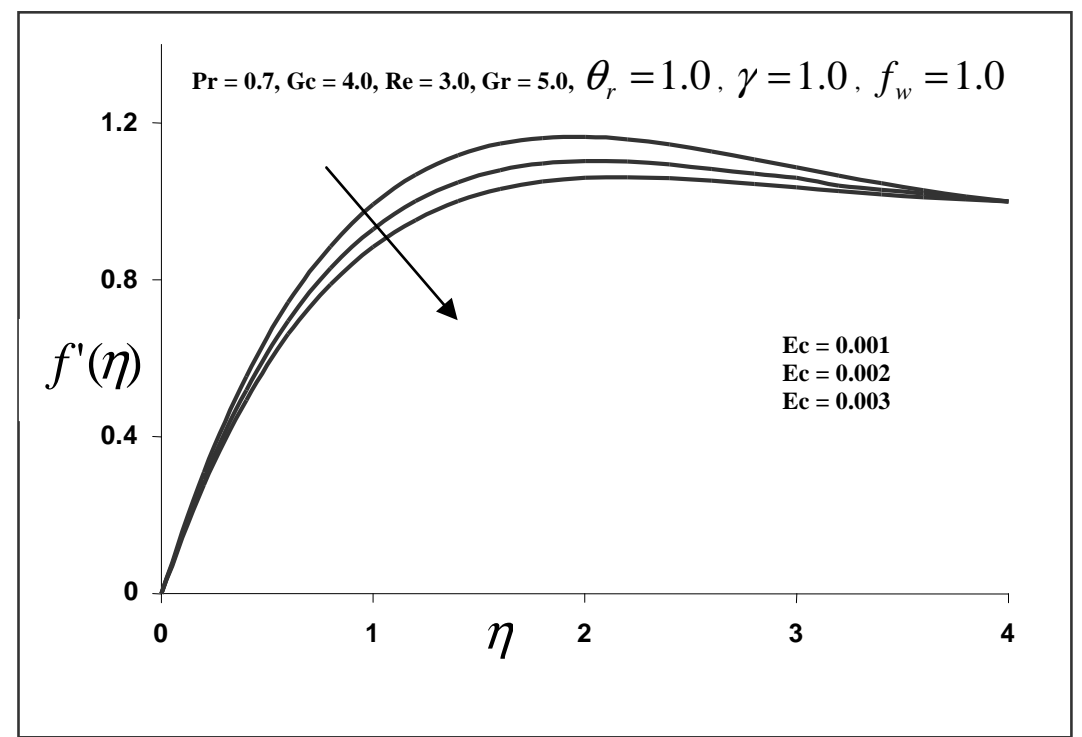

Figure 18 Velocity profiles for different values of Eckert number, Ec

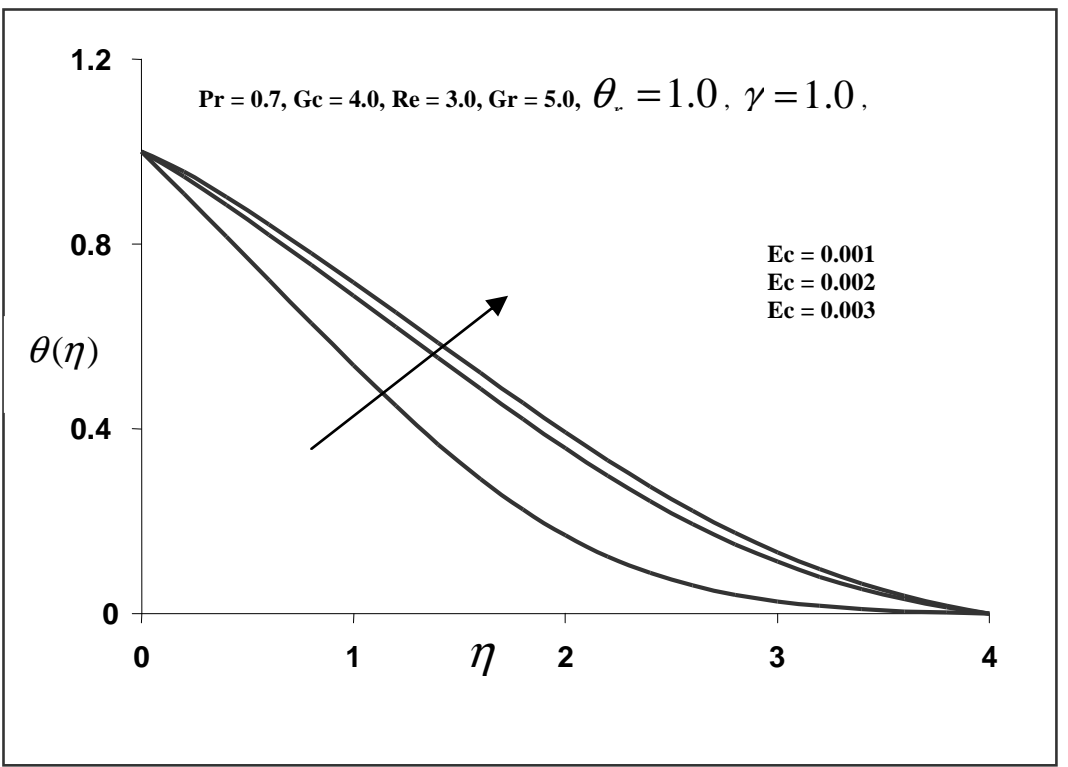

Figure 19 Temperature profiles for different values of Eckert number, Ec 


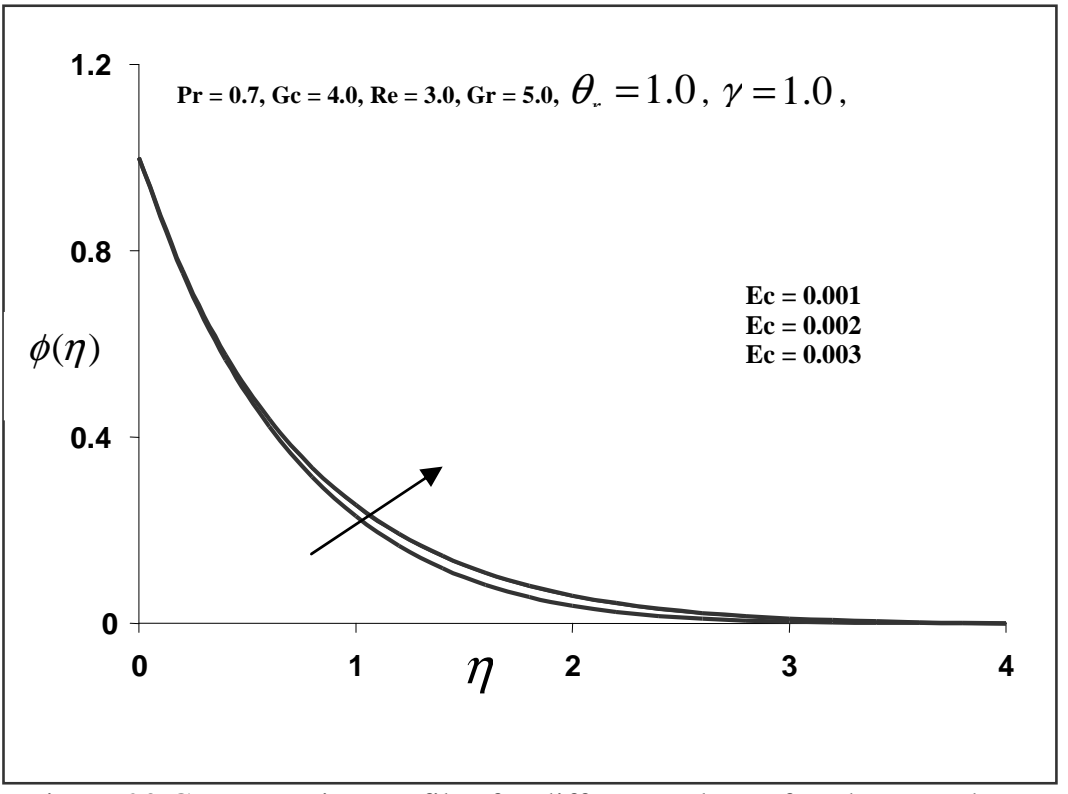

Figure 20 Concentration profiles for different values of Eckert number, Ec

\section{Conclusions}

The effects of viscous dissipation, variable viscosity, suction, and chemical reaction on flow, heat and mass transfer of a steady incompressible Newtonian fluid past a vertical plate have been studied numerically using the implicit finite difference method. From the previous results and discussion, we conclude the following:

- The temperature of the fluid increases with increase in viscous dissipation.

- The velocity increases with the increase of the variable viscosity parameter

- The chemical reaction parameter or the suction parameter decelerates the fluid motion

- The temperature profiles decreases as the viscosity or the suction parameter increases.

- The concentration profiles decreases with the increase the values of chemical reaction or the suction parameter or Reynold number and Schmidt number.

- The skin-friction coefficient, the Nusselt number, and the Sherwood number increase as the viscosity or the suction parameter increases.

- The skin-friction coefficient or the Nusselt number coefficient decrease with the increase of the chemical reaction parameter, but it decreases the Sherwood number coefficient. With the increase of Eckert number the skin-friction coefficient decreases, whereas it increases the Nusselt number coefficient and the Sherwood number coefficient.

\section{References}

Bellman R.E., and Kalaba R.E., Quasi-linearization and Non-Linear Boundary Value Problem, Elsevier, New York. 1965.

Das, S.S., Satapathy, A., Das, J.K., Panda, J.P. 2008. Mixed convection heat and mass transfer in three dimensional flow of a viscous incompressible fluid past a vertical porous plate through a porous medium with periodic permeability, International Journal of Applied Engineering Research, Vol.3, No. 9.

Dessie H., Naikoti K., 2014, MHD effects on heat transfer over stretching sheet embedded in porous medium with variable viscosity, viscous dissipation and heat source/sink, Ain Shams Engineering Journal, Vol., No.3, pp 967-977.

Hakiem M.A., EL- Mohammadeian A.A., El-Kabier S.M.M., Gorla R.S.R., 1999. Joule heating effects on MHD free convection flow of a micro polar fluid, International Journal of Communications in Heat and Mass Transfer, Vol. 26, pp 219-227.

Hassanien I.A., T.H. Al-arabi, 2009. Non-Darcy unsteady mixed convection flow near the stagnation point on a heated vertical surface embedded in a medium with thermal radiation and variable viscosity, Communications in Nonlinear Science and Numerical Simulation, Vol.14, 4, pp 1366-1376.

Hassanien I.A., 1997. The effects of variable viscosity on flow and heat transfer on a continuous stretching surface, ZAMM, Vol. 77, 11, pp $876-880$.

Hossain M.A., .S.Kabir, and D.A.S.Rees, 2002. Natural convection of fluid with variable viscosity from a heated vertical wary surface, ZAMP, Vol. 53, pp 48-52.

Hossian M.A., 1992. Viscous and Joule heating effects on MHD free convection flow with variable plate temperature, International journal of Heat and Mass Transfer, Vol. 35, pp 3485. 
Ishak A., Nazar R., Pop I. 2008. Mixed convection boundary layer flow over a vertical surface embedded in a thermally stratified porous medium, Physics Letters A, Vol. 372, 14, pp 2355-2358.

Jayanthi S. and Kumari M., 2007. Effect of variable viscosity on non-Darcy free or mixed convection flow on a vertical surface in a fluid saturated porous medium, Applied Mathematics and Computations, Vol.186, 2, pp 1643-1659.

Kafoussius N.G. and Williams E. W., 1995. The effect of temperature-dependent viscosity on free forced convective laminar boundary layer flow past a vertical isothermal flat plate," Acta Mechanica, Vol. 110, 1-4, pp 123-137.

Kafoussias N.G., Rees D. A. S., and Daskalakis J. E., 1998. Numerical study of the combined free-forced convective laminar boundary layer flow past a vertical isothermal flat plate with temperature-dependent viscosity, Acta Mechanica, Vol.127, 1-4, pp 39-50.

Kandasamy R., Hashim I., Muhaimin and Ruhaila, 2007. Effects of variable viscosity heat and mass transfer on non-linear mixed convection flow over a porous wedge with heat and radiation in the presence of homogeneous chemical reaction, ARPN Journal of Engineering and Applied Science, B Vol.2, 5, pp 44-53.

Kandasamy, R.; Muhaimin I. Khamis Azme B.' 2009. Thermophoresis and variable viscosity effects on MHD mixed convective heat and mass transfer past a porous wedge in the presence of chemical reaction, Heat and Mass Transfer, Vol. 45, pp 703-712.

Lai F. C. and F.A.Kulacki, 1990. The effect of variable viscosity on convective heat transfer along a vertical surface in a saturated porous medium, International Journal of Heat and Mass Transfer, Vol.33, 5, pp 1028-1031.

Mohamed and Ali, 2006. The effect of variable viscosity on mixed convection heat transfer on a vertical moving surface, International Journal of Thermal Sciences, Vol.45, 1, pp 60 -69.

Mostafa A.A. Mahmoud, 2007. A note on a variable viscosity and chemical reaction effects on mixed convection heat and mass transfer along a semi-infinite vertical plate, Mathematical Problem in Engineering, Vol. 2007, pp.1-7.

Nazar R., Amin N., Pop I., 2004. Unsteady mixed convection boundary layer flow near the stagnation point on a vertical surface in a porous medium, International Journal of Heat and Mass Transfer, Vol.47 No. 12-13, pp.2681-2688.

Pantokratoras A., 2006. The Falkner-Skan flow with constant wall temperature and variable viscosity, International Journal of Thermal Sciences, Vol. 45, pp. 378.

Pop I. and D.B.Ingham, 2001. Convective Heat Transfer, Mathematical and Computational Modeling of Viscous Fluids and Porous Media, Pergamon, London UK,.

Pop I., Gorla R.S.R. and Rashidi M., 1992. The effect of variable Viscosity on flow and heat transfer to a continuous moving flat plate, International Journal of Engineering Science, Vol.30,1, pp 1-5.

\section{Biographical notes}

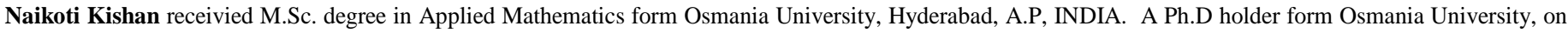

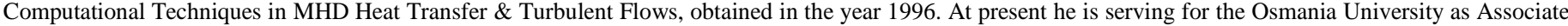

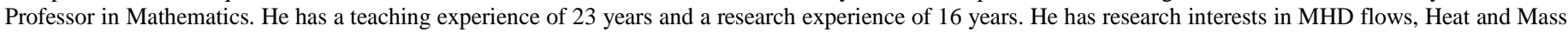

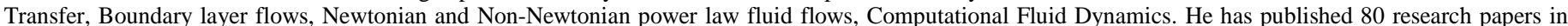

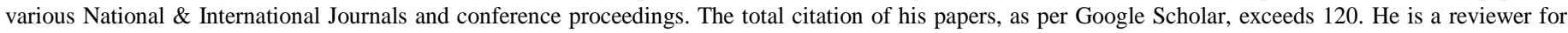

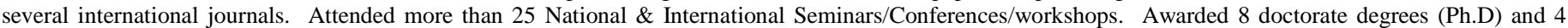
M.Phil degrees. Presently, 8 Ph.D students and 2 M.Phil students are working.

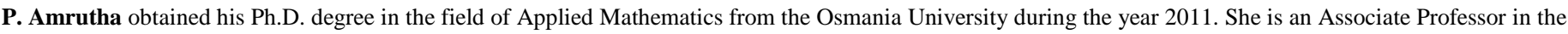

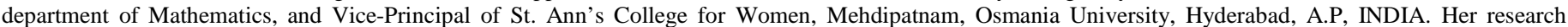

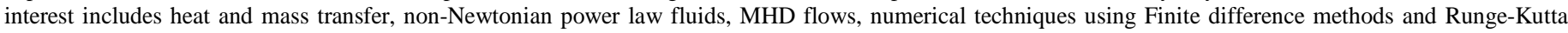
method. Attended more than 10 National \& International Seminars/Conferences/workshops.

Received February 2014

Accepted March 2015

Final acceptance in revised form April 2015 